\title{
Liver cirrhosis leads to poorer survival in patients with end-stage renal disease
}

\author{
Ae Jin Kim, Hye Jin Lim, Han Ro, Ji Yong Jung, Hyun Hee Lee, Wookyung Chung, and Jae Hyun Chang
}

Department of Internal Medicine, Gachon University Gil Medical Center, Graduate School of Medicine, Gachon University, Incheon, Korea

Received: October 28, 2014 Revised : January 20, 2015 Accepted: March 6, 2015

\section{Correspondence to}

Jae Hyun Chang, M.D.

Division of Nephrology,

Department of Internal Medicine,

Gachon University Gil Medical

Center, Graduate School of

Medicine, Gachon University,

21 Namdong-daero 774beon-gil,

Namdong-gu, Incheon 21565,

Korea

Tel: +82-32-460-8923

Fax: +82-32-460-3431

E-mail:jhchang@gilhospital.com
Background/Aims: Liver cirrhosis (LC) is an important problem in patients with end-stage renal disease (ESRD). Few studies have investigated the influence of LC on mortality in patients with ESRD. This study investigated the association between LC and mortality among patients with ESRD and compare mortality between two dialysis modalities.

Methods: Adult patients ( $\geq 18$ years of age) starting dialysis for ESRD were enrolled in the present study from 2000 to 2011 . We analyzed 1,069 patients with ESRD; of these, 742 patients were undergoing hemodialysis (HD) and 327 patients were undergoing peritoneal dialysis (PD).

Results: The prevalence of LC was 44 of 1,069 patients (4.1\%). The cumulative 1-, 3 -, and 5-year survival rates of noncirrhotic patients were $93 \%, 83 \%$, and $73 \%$, respectively, whereas the equivalent survival rates of cirrhotic patients were $90 \%$, $68 \%$, and $48 \%$, respectively $(p=0.011)$. After adjustment, LC was an independent risk factor for death in patients with ESRD. No difference in mortality associated with LC was found between the HD and PD subgroups.

Conclusions: Of the patients with ESRD, cirrhotic patients had poorer survival than noncirrhotic patients. Among patients with ESRD and LC, survival of patients undergoing PD may be comparable with that of patients undergoing HD.

Keywords: Hemodialysis; Liver cirrhosis; Mortality; Peritoneal dialysis

\section{INTRODUCTION}

End-stage renal disease (ESRD) and chronic liver disease are serious and common medical problems worldwide. As the prevalence of ESRD and liver cirrhosis (LC) increase, physicians are likely to be faced with both of these diseases in one patient. The prevalence and incidence of ESRD have been increasing annually worldwide $[1,2]$, based on the growth of the number of patients with diabetes or hypertension. Patients with ESRD are at higher risk of viral hepatitis than are patients among the general population $[3,4]$. The risk of progression to chronic conditions, such as LC and hepatocellular carcinoma, is also higher in these patients than in healthy individuals [4].

The main causes of LC in more developed countries are hepatitis $\mathrm{C}$ virus (HCV) infection, alcohol consumption, and, increasingly, nonalcoholic liver disease. Hepatitis $B$ virus (HBV) infection is the most common cause in sub-Saharan Africa and most parts of Asia. Korea is an endemic area for HBV infection, and the incidence of HBV-related LC is high. Moreover, the increase in the incidence of HCV infection secondary to an increase in the numbers of young drug and alcohol abusers in changing socioeconomic environments emphasizes the clinical importance of LC [5]. The prevalence of patients with combined ESRD and cirrhosis is not well known, but $6.2 \%$ of patients with ESRD reportedly had LC when 
beginning renal replacement therapy in a national cohort study in Taiwan [6]. LC is an increasingly more frequent cause of morbidity and mortality in more developed countries, being the 14th most common cause of death worldwide but the $4^{\text {th }}$ most common in central Europe $[7,8]$. The long-term outcome of LC is associated with complications of liver failure; additionally, renal failure has been identified as a prognostic factor and is associated with high mortality [9].

Although the combined presence of LC and ESRD is clinically important, few studies have compared mortality between patients with and without cirrhosis $[6,10]$, and the optimum modalities of renal replacement therapy for patients with LC remain controversial $[6,11,12]$. Therefore, the aim of this study was to investigate the association between LC and mortality among patients with ESRD and compare mortality between two dialysis modalities.

\section{METHODS}

\section{Study population}

From January 2000 to December 2011, we enrolled patients who began dialysis therapy for ESRD at the Gachon University Gil Medical Center, Korea. Patients were excluded if they had recovered renal function or were younger than 18 years of age. We also excluded those who had undergone renal transplantation during the follow-up period or had incomplete medical records.

All patients' medical records were retrospectively reviewed with respect to age, sex, cause of ESRD, laboratory data, and comorbid conditions at the time of initiation of dialysis. The comorbid conditions included diabetes, cardiovascular disease, chronic obstructive pulmonary disease, systemic vasculitis, dementia, and cancer. We also collected information on the hepatitis serology and liver imaging results of patients with hepatitis. The diagnosis of LC was based on a combination of clinical conditions, such as hepatic encephalopathy, esophageal and gastric varix, and ascites as well as biochemical and radiographic criteria. The causes of LC were evaluated and included HBV or HCV infection, alcoholic liver disease, and other liver diseases. The severity of LC was assessed according to the Child-Pugh classification [13], which considers serum bilirubin, albumin, prothrombin time, ascites, hepatic encephalopathy; patients were designated as class A if the total score was 5 or 6 , class B if the score was 7 to 9 , and class $C$ if the score was 10 or higher. Liver-related death was defined as death associated with LC or its complications, such as esophageal and gastric varices, spontaneous bacterial peritonitis, hepatic failure, and primary liver cancer. Death not directly related to LC was classified as non-liver related death.

The patients were followed from the initiation of dialysis until the end of the study (December 2011) or death. Patients on dialysis during the follow-up period were censored on the date of loss to follow-up. This study was approved by the Institutional Review Board of Gachon University Gil Medical Center. The requirement for informed consent was waived by the board.

\section{Statistical analyses}

Data are presented as mean \pm standard deviation or numbers (\%). Categorical data are expressed as frequency (\%). The Kolmogorov-Smirnov test was used to analyze the normality of the distribution of the parameters measured. Comparisons between the two groups were made by Student $t$ test and the chi-square test. Skewed data were analyzed with the Mann-Whitney $U$ test and Fisher exact test. Mortality was determined using the Kaplan-Meier method and compared using the logrank test. Cox regression analysis was used to assess the risk factors for mortality. Associations are presented as hazard ratios (HRs) with their corresponding 95\% confidence intervals (CIs). All statistical analyses were performed with SPSS version 13.0 (SPSS Inc., Chicago, IL, USA). A value of $p<0.05$ was considered to indicate statistical significance.

\section{RESULTS}

\section{Patients' characteristics}

Demographic and laboratory data are listed in Table 1. We analyzed 1,069 patients with ESRD; of these, 742 were undergoing hemodialysis (HD) and 327 were undergoing peritoneal dialysis (PD). The prevalence of LC was 44 of the 1,069 patients (4.1\%). The LC group was more likely to be younger and male compared with the non- 
Table 1. Characteristics of all patients

\begin{tabular}{|c|c|c|c|c|}
\hline Characteristic & $\begin{array}{c}\text { Total } \\
(\mathrm{n}=1,069)\end{array}$ & $\begin{array}{l}\text { LC group } \\
(\mathrm{n}=44)\end{array}$ & $\begin{array}{l}\text { Non-LC group } \\
(n=1,025)\end{array}$ & $p$ value \\
\hline Age, yr & $55.0 \pm 14.6$ & $50.9 \pm 10.7$ & $55.2 \pm 14.7$ & 0.015 \\
\hline Male sex & $599(56.0)$ & $34(77 \cdot 3)$ & $565(55.1)$ & 0.004 \\
\hline Cause of ESRD & & & & 0.181 \\
\hline Diabetes & $599(56.0)$ & $21(47 \cdot 7)$ & $578(56.4)$ & \\
\hline Hypertension & $90(8.4)$ & $5(11.4)$ & $85(8.3)$ & \\
\hline Glomerulonephritis & $79(7 \cdot 4)$ & $6(13.6)$ & $73(7 \cdot 1)$ & \\
\hline Others & $301(28.2)$ & $12(27 \cdot 3)$ & $289(28.2)$ & \\
\hline Dialysis modality & & & & 0.411 \\
\hline Hemodialysis & $742(69.4)$ & $33(75)$ & $709(69.2)$ & \\
\hline Peritoneal dialysis & $327(30.6)$ & $11(25)$ & $316(30.8)$ & \\
\hline HBsAg positive & $81(7.6)$ & $38(86.4)$ & $43(4 \cdot 2)$ & $<0.001$ \\
\hline Anti-HCV positive & $21(2.0)$ & $1(2.3)$ & $20(2.0)$ & 0.880 \\
\hline \multicolumn{5}{|l|}{ Baseline comorbidity } \\
\hline Diabetes & $626(58.6)$ & $26(59.1)$ & $600(58.5)$ & 0.904 \\
\hline Cardiovascular disease & $367(34 \cdot 3)$ & $10(22.7)$ & $357(34.8)$ & 0.111 \\
\hline COPD & $14(1.3)$ & 0 & $14(1.4)$ & 0.436 \\
\hline Systemic vasculitis & $14(1.3)$ & o & $14(1.4)$ & 0.453 \\
\hline Dementia & $31(2.9)$ & 0 & $31(3.0)$ & 0.250 \\
\hline Cancer & $28(2.6)$ & $3(6.8)$ & $25(2.4)$ & 0.112 \\
\hline \multicolumn{5}{|l|}{ Laboratory } \\
\hline Hemoglobin, g/dL & $8.4 \pm 1.7$ & $8.1 \pm 1.5$ & $8.4 \pm 1.7$ & 0.244 \\
\hline Creatinine, mg/dL & $8.0 \pm 4.0$ & $8.0 \pm 4.5$ & $8.0 \pm 4.0$ & $>0.999$ \\
\hline Total protein, g/dL & $6.1 \pm 0.9$ & $6.2 \pm 0.7$ & $6.1 \pm 0.9$ & 0.662 \\
\hline Albumin, g/dL & $3.2 \pm 0.6$ & $3.0 \pm 0.5$ & $3 \cdot 3 \pm 0.6$ & 0.020 \\
\hline Total bilirubin, mg/dL & $0.5 \pm 0.5$ & $0.6 \pm 0.4$ & $0.5 \pm 0.5$ & 0.085 \\
\hline AST, U/L & $30 \pm 134$ & $30 \pm 19$ & $30 \pm 137$ & 0.971 \\
\hline ALT, U/L & $24 \pm 112$ & $25 \pm 19$ & $24 \pm 114$ & 0.427 \\
\hline INR & $1.03 \pm 0.25$ & $1.10 \pm 0.17$ & $1.02 \pm 0.25$ & 0.057 \\
\hline Total cholesterol, mg/dL & $161 \pm 46$ & $149 \pm 37$ & $161 \pm 47$ & 0.138 \\
\hline $\mathrm{Ca} \times \mathrm{P}, \mathrm{mg}^{2} / \mathrm{dL}^{2}$ & $43.2 \pm 16.7$ & $42.3 \pm 16.6$ & $43.2 \pm 16.7$ & 0.722 \\
\hline
\end{tabular}

Values are presented as mean $\pm \mathrm{SD}$ or number (\%).

LC, liver cirrhosis; ESRD, end-stage renal disease; HBsAg, hepatitis B surface antigen; HCV, hepatitis C virus; COPD, chronic obstructive pulmonary disease; AST, aspartate aminotransferase; ALT, alanine aminotransferase; INR, international normalized ratio; $\mathrm{Ca} \times \mathrm{P}$, calcium phosphorus product.

LC group. Cirrhotic patients had a significantly higher prevalence of HBV infection, which was a major cause of LC. Cirrhotic patients had a significantly lower serum albumin level, but other laboratory data showed no difference between the LC and non-LC groups. There were no significant differences in the causes of ESRD, the dialysis modality, or the baseline comorbidities.

\section{Liver cirrhosis and mortality in patients with ESRD}

During the follow-up period, 213 patients (19.9\%) died. The mean follow-up duration of all patients was $38.0 \pm$ 33.6 months (interquartile range, 10 to 56 ). The mean fol- 
low-up time alive on dialysis in the LC group was 35.0 \pm 32.7 months, and that in the non-LC group was $38.0 \pm$ 33.7 months. The cumulative 1-, 3-, and 5-year survival rates of all patients were $93 \%, 82 \%$, and $72 \%$, respectively. Patients with concurrent ESRD and LC had a higher mortality rate than did patients with ESRD without LC ( $p=0.011)$. The cumulative 1-, 3-, and 5-year survival rates of noncirrhotic patients were $93 \%, 83 \%$, and $73 \%$, respectively. The cumulative 1-, 3-, and 5-year survival rates of cirrhotic patients were $90 \%, 68 \%$, and $48 \%$, respectively (Fig. 1). Cirrhotic patients had higher liver-related mortality than that of noncirrhotic patients. The most com-

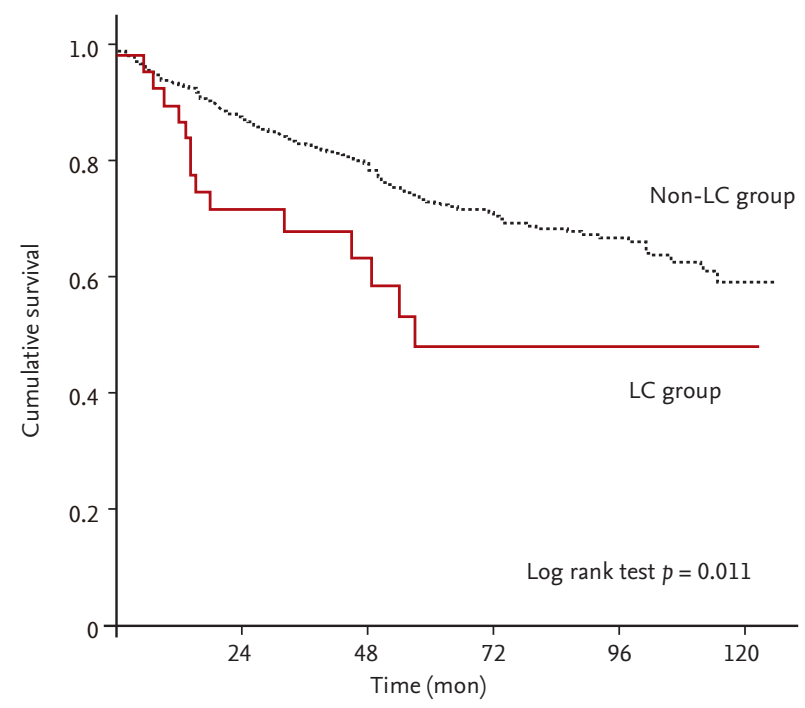

Figure 1. All-cause mortality of noncirrhotic and cirrhotic patients with end-stage renal disease. Patients with liver cirrhosis (LC) had a higher mortality rate than those without LC $(p=0.011)$. mon causes of death in the LC group were liver-related and cardiovascular. However, the most common causes of death in the non-LC group were cardiovascular and infection (Table 2).

We used Cox proportional regression analyses to evaluate the possible risk factors for all-cause mortality. Univariate analysis demonstrated that $\mathrm{LC}$ was associated with mortality (HR, 1.956; 95\% CI, 1.157 to 3.307; $p=0.011$ ). Age, diabetes, cardiovascular disease, serum albumin of $<3.5 \mathrm{mg} / \mathrm{dL}$, total bilirubin, and the international normalized ratio (INR) were also associated with mortality (Table 3). Multivariate Cox proportional hazards analyses revealed that LC remained a significant predictor of mortality even after adjustments for age (model 1: HR, 2.595; 95\% CI, 1.524 to 4.420; $p<0.001$ ); age, albumin of < $3.5 \mathrm{mg} / \mathrm{dL}$, and INR (model 2: HR, 2.218; 95\% CI, 1.278 to 3.848; $p=0.005$ ); age, diabetes, and cardiovascular disease (model 3: HR, 2.703; 95\% CI, 1.585 to 4.609; $p<0.001$ ); and age, albumin of $<3.5 \mathrm{mg} / \mathrm{dL}$, INR, total bilirubin, diabetes, and cardiovascular disease (model 4: HR, 2.172; 95\% CI, 1.225 to $3.851 ; p=0.008$ ) (Table 4 ).

\section{Risk factors for mortality in cirrhotic patients with ESRD}

The causes of LC were HBV infection (75.0\%), HCV infection (2.2\%), alcohol consumption (11.4\%), and HBV infection combined with alcohol consumption (11.4\%). In total, 21 patients were classified as Child class A, 16 as class B, and 7 as class C. Esophageal or gastric varices were found in six patients (13.6\%), ascites was found in 12 patients (27.3\%), and hepatic encephalopathy was

Table 2. Causes of death

\begin{tabular}{|c|c|c|c|}
\hline Variable & LC group $($ death $=16)$ & Non-LC group $($ death $=197)$ & $p$ value \\
\hline Liver related & 4 & 0 & $<0.001$ \\
\hline Varix bleeding & 2 & o & \\
\hline Hepatocellular carcinoma & 1 & o & \\
\hline Spontaneous bacterial peritonitis & 1 & 0 & \\
\hline Non-liver related & 8 & 138 & $0.15^{8}$ \\
\hline Cardiovascular disease & 4 & 62 & \\
\hline Infection & 1 & 62 & \\
\hline Malignancy & o & 1 & \\
\hline Others & 3 & 13 & \\
\hline Unknown & 4 & 59 & 0.783 \\
\hline
\end{tabular}

LC, liver cirrhosis. 
Table 3. Hazard ratios and $95 \%$ confidence intervals for mortality by univariate Cox proportional hazards analysis

\begin{tabular}{lccc}
\hline Variable & HR & $95 \%$ CI & p value \\
\hline Liver cirrhosis & 1.956 & $1.157-3.307$ & 0.011 \\
Age, yr & 1.043 & $1.032-1.054$ & $<0.001$ \\
Diabetes & 1.733 & $1.299-2.311$ & $<0.001$ \\
Cardiovascular disease & 1.491 & $1.130-1.966$ & 0.005 \\
Hemoglobin $<10 \mathrm{~g} / \mathrm{dL}$ & 1.032 & $0.747-1.425$ & 0.848 \\
Serum albumin $<3.5 \mathrm{~g} / \mathrm{dL}$ & 1.733 & $1.281-2.344$ & $<0.001$ \\
Total bilirubin, $\mathrm{mg} / \mathrm{dL}$ & 1.299 & $1.079-1.565$ & 0.006 \\
AST $>40 \mathrm{U} / \mathrm{L}$ & 1.330 & $0.819-2.160$ & 0.249 \\
ALT $>40 \mathrm{U} / \mathrm{L}$ & 1.269 & $0.782-2.060$ & 0.335 \\
INR & 2.098 & $1.451-3.034$ & $<0.001$ \\
\hline
\end{tabular}

HR, hazard ratio; CI, confidence interval; AST, aspartate aminotransferase; ALT, alanine aminotransferase; INR, international normalized ratio.

Table 4. Hazard ratios and $95 \%$ confidence intervals for mortality by multivariate Cox proportional hazards analysis

\begin{tabular}{lcrr}
\hline \multirow{2}{*}{ Variable } & \multicolumn{3}{c}{ Liver cirrhosis } \\
\cline { 2 - 4 } & HR & $95 \%$ CI & $p$ value \\
\hline Model 1 $^{\mathrm{a}}$ & 2.595 & $1.524-4.420$ & $<0.001$ \\
Model $^{\mathrm{b}}$ & 2.218 & $1.278-3.848$ & 0.005 \\
Model $^{\mathrm{c}}$ & 2.703 & $1.585-4.609$ & $<0.001$ \\
Model $^{\mathrm{d}}$ & 2.172 & $1.225-3.851$ & 0.008 \\
\hline
\end{tabular}

HR, hazard ratio; CI, confidence interval.

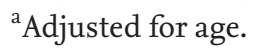

${ }^{\mathrm{b}}$ Adjusted for age, albumin $<3.5 \mathrm{~g} / \mathrm{dL}$, and the international normalized ratio (INR).

${ }^{\mathrm{c}}$ Adjusted for age, diabetes, and cardiovascular disease.

${ }^{\mathrm{d}}$ Adjusted for age, albumin <3.5 g/dL, INR, total bilirubin, diabetes, and cardiovascular disease.

found in six patients (13.6\%). The initial dialysis modalities were $\mathrm{HD}$ in 33 patients and PD in 11 patients. The prevalence of LC was $4.4 \%$ and $3.6 \%$ in the HD and PD groups, respectively. No significant differences were observed in the baseline characteristics of cirrhotic patients based on dialysis modality (Table 5).

We used Cox proportional regression analyses to evaluate possible risk factors for all-cause mortality in cirrhotic patients with ESRD. The univariate analysis demonstrated that total bilirubin (HR, 6.246; 95\% CI, 2.078 to $18.775 ; p=0.001$ ), Child-Pugh classification (HR, 2.362; $95 \% \mathrm{CI}, 1.187$ to $4.700 ; p=0.014$ ), and diabetes (HR, 3.496; $95 \%$ CI, 1.022 to $11.961 ; p=0.046)$ were associated with mortality. No significant difference in survival was observed according to dialysis modality in cirrhotic patients (Fig. 2). After adjusting for confounding variables, total bilirubin (HR, 9.139; 95\% CI, 2.054 to 40.659; $p=0.004$ ) and diabetes (HR, 5.531; 95\% CI, 1.201 to 25.473; $p=0.028)$ were significant risk factors for mortality in patients with ESRD and LC.

\section{DISCUSSION}

This study investigated the long-term survival, and mortality risk of LC among patients undergoing dialysis and the effect of dialysis modality on the outcome of cirrhotic patients. We showed that patients with concurrent ESRD and LC had a significantly lower survival rate than did noncirrhotic patients, and LC was an in- 
Table 5. Characteristics of the cirrhotic patients

\begin{tabular}{|c|c|c|c|c|}
\hline Characteristic & $\operatorname{Total}(\mathrm{n}=44)$ & Hemodialysis $(\mathrm{n}=33)$ & Peritoneal dialysis $(\mathrm{n}=11)$ & $p$ value \\
\hline Age, yr & $50.9 \pm 10.7$ & $50.3 \pm 11.4$ & $52.6 \pm 8.6$ & 0.541 \\
\hline Male sex & $34(77 \cdot 3)$ & $27(81.8)$ & $7(63.6)$ & 0.237 \\
\hline Diabetes & $26(59.1)$ & $20(60.6)$ & $6(54.5)$ & 0.723 \\
\hline Cardiovascular disease & $10(22.7)$ & $7(21.2)$ & $3(27.3)$ & 0.692 \\
\hline Cause of liver cirrhosis & & & & 0.153 \\
\hline HBV infection & $33(75)$ & $25(75.8)$ & $8(72.7)$ & \\
\hline Alcohol & $5(11.4)$ & $5(15.2)$ & o & \\
\hline HCV infection & $1(2.2)$ & o & $1(9.1)$ & \\
\hline Alcohol and HBV infection & $5(11.4)$ & $3(9.1)$ & $2(18.2)$ & \\
\hline Child-Pugh class & & & & 0.770 \\
\hline A & $21(47.7)$ & $15(45 \cdot 5)$ & $6(54 \cdot 5)$ & \\
\hline B & $16(36.4)$ & $13(39.4)$ & $3(27.3)$ & \\
\hline $\mathrm{C}$ & $7(15.9)$ & $5(15.1)$ & $2(18.2)$ & \\
\hline Varix (esophageal, gastric) & $6(13.6)$ & $6(18.2)$ & o & 0.311 \\
\hline Ascites & $12(27.3)$ & $11(33 \cdot 3)$ & $1(9.1)$ & 0.240 \\
\hline Hepatic encephalopathy & $6(13.6)$ & $6(18.2)$ & o & 0.311 \\
\hline \multicolumn{5}{|l|}{ Laboratory } \\
\hline Hemoglobin, g/dL & $8.1 \pm 1.5$ & $8.1 \pm 1.5$ & $8.4 \pm 1.4$ & 0.522 \\
\hline Creatinine, mg/dL & $8.0 \pm 4.5$ & $8.2 \pm 5.0$ & $7.5 \pm 2.8$ & 0.684 \\
\hline Total protein, g/dL & $6.2 \pm 0.7$ & $6.2 \pm 0.7$ & $6.2 \pm 0.8$ & 0.864 \\
\hline Albumin, g/dL & $3.0 \pm 0.5$ & $3.0 \pm 0.5$ & $3.0 \pm 0.7$ & 0.833 \\
\hline Total bilirubin, mg/dL & $0.6 \pm 0.4$ & $0.6 \pm 0.4$ & $0.6 \pm 0.4$ & 0.963 \\
\hline AST, U/L & $30 \pm 19$ & $30 \pm 16$ & $34 \pm 26$ & 0.557 \\
\hline ALT, U/L & $25 \pm 19$ & $26 \pm 19$ & $22 \pm 23$ & 0.529 \\
\hline INR & $1.10 \pm 0.17$ & $1.11 \pm 0.18$ & $1.06 \pm 0.12$ & 0.410 \\
\hline Total cholesterol, mg/dL & $149 \pm 37$ & $147 \pm 38$ & $154 \pm 36$ & 0.138 \\
\hline $\mathrm{Ca} \times \mathrm{P}, \mathrm{mg}^{2} / \mathrm{dL}^{2}$ & $42.3 \pm 16.6$ & $42.6 \pm 15.0$ & $41.2 \pm 22.0$ & 0.813 \\
\hline
\end{tabular}

Values are presented as mean $\pm \mathrm{SD}$ or number (\%).

HBV, hepatitis B virus; HCV, hepatitis C virus; AST, aspartate aminotransferase; ALT, alanine aminotransferase; INR, international normalized ratio; $\mathrm{Ca} \times \mathrm{P}$, calcium phosphorus product.

dependent predictor of mortality. There was no significant survival difference between HD and PD in cirrhotic patients.

In our study, the prevalence of LC in patients with ESRD was $4.1 \%$. The prevalence of cirrhosis among patients with ESRD is not well known, but $4 \%$ to $6 \%$ of patients with ESRD have been found to have LC when beginning renal replacement therapy $[6,10,14]$. The main cause of liver disease is viral hepatitis. The Asia-Pacific area is highly endemic for viral hepatitis infection among patients undergoing dialysis [15], but the preva- lence of viral hepatitis differs according to the geographical area. Korea is an endemic area for HBV infection, while Taiwan is an endemic area for HCV infection. Approximately $4.4 \%$ of patients undergoing $\mathrm{HD}$ and $3.6 \%$ of those undergoing PD have LC. Patients undergoing HD have a higher rate of LC than those undergoing PD. As shown by our results, many more patients undergoing HD than PD had LC in a national cohort study in Taiwan (6.2\% vs. $5.3 \%$, respectively) [6].

Of the patients with ESRD, those with cirrhosis had poorer survival than those without cirrhosis. Cirrho- 


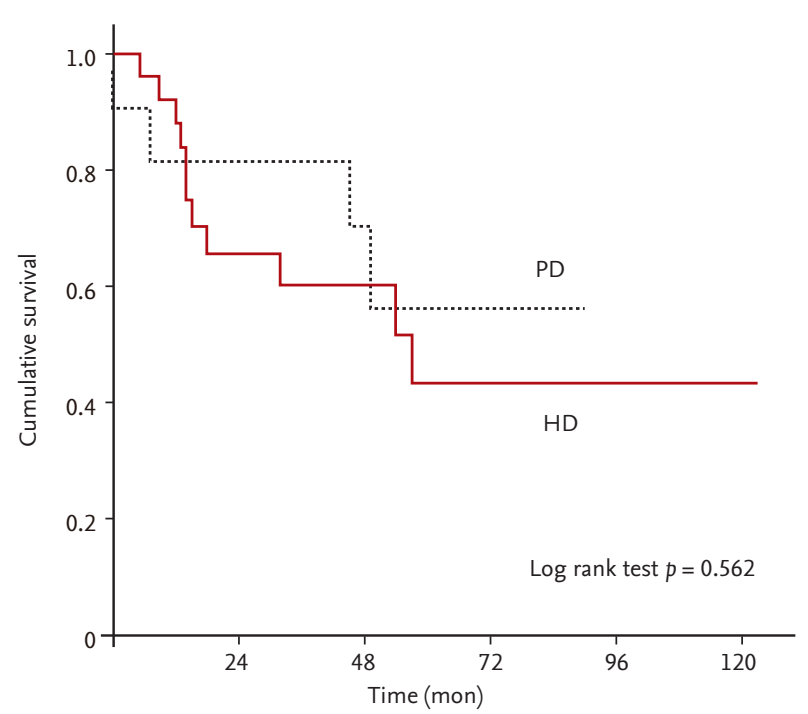

Figure 2. Survival of cirrhotic patients undergoing hemodialysis (HD) and peritoneal dialysis (PD). No difference in survival was observed according to dialysis modality in cirrhotic patients $(p=0.562)$.

sis is an increasingly frequent cause of morbidity and mortality. LC was an important predictor of mortality in patients undergoing dialysis in a cohort study in Taiwan [6]. No laboratory data were evaluated in that study. Comorbidities such as diabetes and cardiovascular disease were identified as risk factors of mortality in patients with ESRD. The mortality of cirrhotic patients with ESRD was confounded by these comorbid conditions. In our study, univariate analysis showed that laboratory values, such as serum albumin of $<3.5 \mathrm{mg} / \mathrm{dL}$, total bilirubin, and INR, were associated with mortality. Therefore, we adjusted for not only comorbidities such as diabetes and cardiovascular disease, but also laboratory values such as albumin, total bilirubin, and INR. After adjustment, LC was an independent risk factor for death in patients with ESRD. The cirrhotic patients had higher liver-related mortality than that of noncirrhotic patients.

The outcome of patients with cirrhosis is associated with the severity and complications of liver failure [16]. The most common prognostic variable in patients with cirrhosis was the Child-Pugh classification or its components (albumin, bilirubin, ascites, encephalopathy, and prothrombin time) [16]. In our study, total bilirubin was related with mortality in cirrhotic patients with ESRD. Child-Pugh classification was associated with survival in the univariate analysis, but was not associated with survival after adjustment. De Vecchi et al. [17] also showed no relationship between survival and Child-Pugh classification in cirrhotic patients undergoing PD. This discrepancy may be explained by the small number of patients. One study showed that diabetes is an independent prognostic factor in patients with cirrhosis [18,19]. This finding is consistent with our results, although the characteristics of the study populations were different.

The most optimal renal replacement therapy for patients with ESRD and concomitant LC remains controversial. We analyzed the effect of dialysis modality on the outcome of cirrhotic patients with ESRD. Our data showed no significant difference in the outcome of LC in patients with ESRD between those undergoing HD and PD. Chien et al. [6] reported no significant difference in survival time according to dialysis modality in patients with ESRD and preexisting LC. In their study, patients undergoing PD had a better crude survival rate than did patients undergoing HD. Compared with patients undergoing $\mathrm{HD}$, those undergoing PD were younger and less likely to have comorbidities. After adjustment, there was no significant difference in survival between the HD and PD groups. Kim et al. [20] also showed no difference in mortality between cirrhotic patients with ESRD undergoing HD and PD.

The initial dialysis modality choice in patients with LC is difficult to determine. PD has several advantages over $\mathrm{HD}$ in patients with $\mathrm{LC}$, as it reduces the risk of HBV and HCV transmission. PD is also hemodynamically more stable than HD in patients with LC because of slow and continuous solute clearance [21]. Direct drainage of ascites via the peritoneal catheter controls the volume of ascites and avoids repeated paracentesis. In addition, PD avoids the need for anticoagulation therapy. Despite these benefits, there are several limitations to the application of PD in cirrhotic patients. The instillation of dialysate may increase the abdominal pressure, which might cause complications of leakage, hydrothorax, and inguinal hernia [10]. To avoid severe abdominal distension, a reduced volume of dialysate may be used, but this could increase the risk of inadequate dialysis. Cirrhotic patients are susceptible to spontaneous bacterial peritonitis [22]. Protein loss into the dialysate may worsen hypoalbuminemia and induce malnutrition in cirrhotic patients. 
HD in patients with LC has several limitations. Because of the decreased peripheral vascular resistance and high circulating nitric oxide levels in these patients [23], the effective arterial blood volume is unavoidably reduced. Cirrhotic patients have a spontaneous tendency to develop arterial hypotension during HD, which limits ultrafiltration and worsens ascites. The increased bleeding tendency due to alterations in clotting factors and platelet abnormalities in cirrhotic patients may also be problematic for those undergoing HD, particularly with arteriovenous access. Rapid osmotic and electrolyte shifts during intermittent HD may potentially aggravate hepatic encephalopathy $[24,25]$. This study had several limitations. First, it was a single-center study, resulting in potential bias. Second, the number of cirrhotic patients was relatively small due to the low prevalence of LC in the dialysis population. Because the number of cirrhotic patients treated with PD is limited, it is difficult to compare outcomes between HD and PD in patients with concurrent ESRD and LC. Finally, there might be some residual confounding factors as in other observational studies; thus, we can show only an association between these risk factors and mortality, not causality.

In conclusion, of the patients with ESRD, cirrhotic patients had poorer survival than noncirrhotic patients. There is no significant survival difference between HD and PD in cirrhotic patients. The choice of modality for renal replacement therapy in cirrhotic patients should be individualized with consideration of conventional indications and complications of LC. Further larger-scale and multicenter studies are needed to elucidate the associations of various risk factors with outcomes and mortality rates in patients with concurrent ESRD and LC.

\section{KEY MESSAGE}

1. Of patients with end-stage renal disease (ESRD), cirrhotic patients had poorer survival than noncirrhotic patients.

2. Survival of patients undergoing peritoneal dialysis was comparable with that of patients undergoing hemodialysis among patients with ESRD and concomitant liver cirrhosis.

\section{Conflict of interest}

No potential conflict of interest relevant to this article was reported.

\section{REFERENCES}

1. Collins AJ, Foley RN, Chavers B, et al. United States Renal Data System 2011 Annual Data Report: atlas of chronic kidney disease \& end-stage renal disease in the United States. Am J Kidney Dis 2012;59(1 Suppl 1):A7, e1-e42O.

2. Jin DC. Current status of dialysis therapy in Korea. Korean J Intern Med 2011;26:123-131.

3. Patel PR, Thompson ND, Kallen AJ, Arduino MJ. Epidemiology, surveillance, and prevention of hepatitis $C$ virus infections in hemodialysis patients. Am J Kidney Dis 2010;56:371-378.

4. Edey M, Barraclough K, Johnson DW. Review article: hepatitis B and dialysis. Nephrology (Carlton) 2010;15:137145 .

5. Han YS, Kim BH, Baek IY, et al. The change of the etiology, complications and cause of death of the liver cirrhosis in 1990s. Korean J Hepatol 2000;6:328-339.

6. Chien CC, Wang JJ, Sun YM, et al. Long-term survival and predictors for mortality among dialysis patients in an endemic area for chronic liver disease: a national cohort study in Taiwan. BMC Nephrol 2012;13:43.

7. Lozano R, Naghavi M, Foreman K, et al. Global and regional mortality from 235 causes of death for 20 age groups in 1990 and 2010: a systematic analysis for the Global Burden of Disease Study 2010. Lancet 2012;380:2095-2128.

8. Blachier M, Leleu H, Peck-Radosavljevic M, Valla DC, Roudot-Thoraval F. The burden of liver disease in Europe: a review of available epidemiological data. J Hepatol 2013;58:593-608.

9. Fede G, D'Amico G, Arvaniti V, et al. Renal failure and cirrhosis: a systematic review of mortality and prognosis. J Hepatol 2012;56:810-818.

10. Huang ST, Chuang YW, Cheng CH, et al. Outcome of peritoneal dialysis in cirrhotic patients with end-stage renal disease: a 24-years' experience in Taiwan. Clin Nephrol 2011;76:306-313.

11. Chaudhary K, Khanna R. Renal replacement therapy in end-stage renal disease patients with chronic liver disease and ascites: role of peritoneal dialysis. Perit Dial Int 
2008;28:113-117.

12. Chaudhary $\mathrm{K}$. What is the best chronic dialysis modality for ESRD patients with end-stage liver disease? Semin Dial 2011;24:414-415.

13. Pugh RN, Murray-Lyon IM, Dawson JL, Pietroni MC, Williams R. Transection of the oesophagus for bleeding oesophageal varices. Br J Surg 1973;60:646-649.

14. Hwang SJ, Yang WC, Lin MY, Mau LW, Chen HC; Taiwan Society of Nephrology. Impact of the clinical conditions at dialysis initiation on mortality in incident haemodialysis patients: a national cohort study in Taiwan. Nephrol Dial Transplant 2010;25:2616-2624.

15. Johnson DW, Dent H, Yao Q, et al. Frequencies of hepatitis $\mathrm{B}$ and $\mathrm{C}$ infections among haemodialysis and peritoneal dialysis patients in Asia-Pacific countries: analysis of registry data. Nephrol Dial Transplant 2009;24:1598-1603.

16. D'Amico G, Garcia-Tsao G, Pagliaro L. Natural history and prognostic indicators of survival in cirrhosis: a systematic review of 118 studies. J Hepatol 2006;44:217-231.

17. De Vecchi AF, Colucci P, Salerno F, Scalamogna A, Ponticelli C. Outcome of peritoneal dialysis in cirrhotic patients with chronic renal failure. Am J Kidney Dis 2002;40:161-168.

18. Hsiang JC, Gane EJ, Bai WW, Gerred SJ. Type 2 diabetes: a risk factor for liver mortality and complications in hepatitis B cirrhosis patients. J Gastroenterol Hepatol 2015;30:591-599.

19. Elkrief L, Chouinard P, Bendersky N, et al. Diabetes mellitus is an independent prognostic factor for major liver-related outcomes in patients with cirrhosis and chronic hepatitis C. Hepatology 2014;60:823-831.

20. Kim MS, Yang JW, Han ST, et al. Comparison between hemodialysis and peritoneal dialysis in patients with endstage renal disease and liver cirrhosis. Korean J Nephrol 2007;26:705-715.

21. Marcus RG, Messana J, Swartz R. Peritoneal dialysis in end-stage renal disease patients with preexisting chronic liver disease and ascites. Am J Med 1992;93:35-40.

22. Such J, Runyon BA. Spontaneous bacterial peritonitis. Clin Infect Dis 1998;27:669-674.

23. Martin PY, Gines P, Schrier RW. Nitric oxide as a mediator of hemodynamic abnormalities and sodium and water retention in cirrhosis. N Engl J Med 1998;339:533-541.

24. Winney RJ, Kean DM, Best JJ, Smith MA. Changes in brain water with haemodialysis. Lancet 1986;2:1107-1108.

25. Donovan JP, Schafer DF, Shaw BW Jr, Sorrell MF. Cerebral oedema and increased intracranial pressure in chronic liver disease. Lancet 1998;351:719-721. 